\title{
The absorption of light by rough metal surfaces-A three-dimensional ray-tracing analysis
}

\author{
D. Bergström, ${ }^{1,2, a)}$ J. Powell, ${ }^{2,3}$ and A. F.H. Kaplan ${ }^{2}$ \\ ${ }_{1}^{1}$ Department of Engineering, Physics and Mathematics, Mid Sweden University, \\ S-831 25 Östersund, Sweden \\ ${ }^{2}$ Division of Manufacturing Systems Engineering, Luleå University of Technology, \\ S-971 87 Luleå, Sweden \\ ${ }^{3}$ Laser Expertise, Ltd., Nottingham NG7 2TR, United Kingdom
}

(Received 5 February 2008; accepted 28 March 2008; published online 22 May 2008)

\begin{abstract}
The laser absorptance of rough surfaces has been investigated by using Monte Carlo simulations based on three-dimensional (3D) ray tracing. The influence of multiple scattering, shadowing, and the Fresnel-equation based angle dependence is discussed. The $3 \mathrm{D}$ results are compared to previously published results from a two-dimensional ray-tracing analysis and the different applications of the two models are explained. (C) 2008 American Institute of Physics.
\end{abstract}

[DOI: $10.1063 / 1.2930808]$

\section{INTRODUCTION}

In laser-material processing, an understanding of the various mechanisms for light absorption is important for the modeling of particular applications (such as welding, cutting, surface treatment, etc.) as well as for optimizing the use of the applied laser energy. The energy of the laser beam is converted into heat in the workpiece through the process of light absorption. In metals, light is absorbed mainly via free electrons (intraband absorption) and/or bound electrons (interband absorption). On a macroscopic level, we can define the absorptance [the convention used here follows that of NIST; for radiative properties the -ivity ending is reserved for pure and smooth surfaces and the -ance ending for all others (i.e., all real surfaces)] as the ratio of the absorbed laser power to the incident laser power. This ratio depends on several parameters including laser wavelength, angle of incidence, polarization, metal oxide layer thickness and structure, surface contamination, and surface roughness. ${ }^{1}$

In a previous study, two-dimensional (2D) ray tracing involving one-dimensional (1D) surface profiles was used to investigate the influence of surface topography on light absorption. ${ }^{2}$ In the present paper, this method has been extended to a full three-dimensional (3D) analysis on 2D rough surfaces. Ray tracing, also known as the geometric optics (GO) approximation in scattering theory, ${ }^{3,4}$ is a common tool in various scientific and engineering applications where the scattering properties of light are of interest, e.g., in computer graphics, optical instrument design, communications, remote sensing, heat transfer analysis, etc. ${ }^{5-7}$ In the GO approximation, energy bundles are traced throughout their interactions with the surface until they leave it. At each interaction point, the directions and amplitudes of the individual energy bundles or rays are determined from Snell's law of reflection and Fresnel's equations, respectively.

For 1D surfaces, which are associated with 2D scatter-

\footnotetext{
${ }^{a)}$ Author to whom correspondence should be addressed. Electronic mail: david.bergstrom@miun.se.
}

ing, ray tracing has been verified by Tang et al. ${ }^{3}$ to be a good approximation to the more exact electromagnetic wave theoretical models based on Maxwell's equations. The authors demonstrated that the approximation is valid as long as $\sigma \cos \left(\theta_{0}\right) / \lambda>0.17$ ( $\sigma$ being the rms roughness, $\lambda$ representing the wavelength of light, and $\theta_{0}$ the angle of incidence, where $\theta_{0}=0^{\circ}$ means normal incidence) and for surfaces with $\sigma / \tau<2.0$ (where $\tau$ is the surface correlation length). Although no rigorous studies of the regions of validity for 3D ray tracing (on 2D surfaces) have been conducted, comparisons made to the few existing results from wave theory and to various experimental results seem to indicate that the approximation is valid for the same range as in the $2 \mathrm{D}$ case. ${ }^{4}$

Section II presents some basic and useful quantities from a radiative theory and describes the generation and properties of the Gaussian random rough surfaces as well as providing a detailed description of the ray-tracing model.

\section{THEORY AND ANALYSIS}

\section{A. Radiative properties}

When light is incident on a material surface, the energy will either be reflected, transmitted, or absorbed. To describe and quantify the angular distribution of the reflected or scattered light, the bidirectional reflection distribution function (BRDF) is defined as ${ }^{8}$

$$
\rho_{\lambda}^{\prime \prime}\left(\Omega_{s}, \Omega_{i}\right)=\frac{\pi}{\cos \left(\theta_{s}\right)}\left(\frac{d \Phi_{s}}{d \Omega_{s}} / \frac{d \Phi_{i}}{d \Omega_{i}}\right),
$$

where $\theta_{s}$ is the polar (zenith) angle of the scattered light, $\Phi_{i}$ and $\Phi_{s}$ are the incident and scattered radiant powers, and $\Omega_{i}\left(\theta_{i}, \varphi_{i}\right)$ and $\Omega_{s}\left(\theta_{s}, \varphi_{s}\right)$ are the incident and scattered solid angles, respectively (see Fig. 1). The BRDF is a fundamental quantity in radiative theory, from which all reflective properties can be derived and is the quantity that is most often derived in scattering models.

Integration of the bidirectional reflectance over the entire hemisphere yields the directional-hemispherical reflectance ${ }^{8}$ 


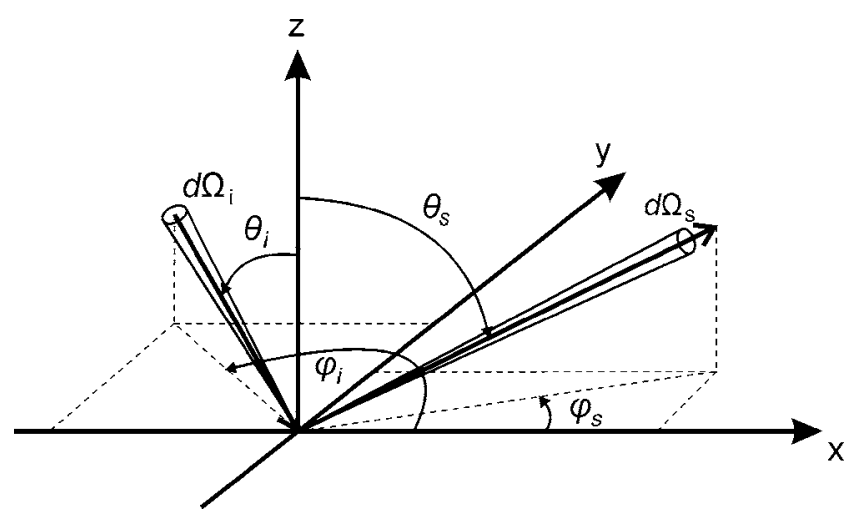

FIG. 1. Definition of the BRDF in a global reference frame. The $x-y$ plane here defines the mean plane of the rough surface and the $z$ axis is normal to this plane. The directions of the incident (subscript $i$ ) and scattered (subscript $s$ ) rays of light, subtended by the solid angles $d \Omega$, are specified by the zenith and the azimuth angles $\theta$ and $\varphi$ respectively.

$$
\rho_{\lambda}^{\prime}\left(\Omega_{i}\right)=\frac{1}{\pi} \int_{2 \pi} \rho_{\lambda}^{\prime \prime}\left(\Omega_{s}, \Omega_{i}\right) \cos \left(\theta_{s}\right) d \Omega_{s}
$$

For opaque materials such as metals (except when dealing with extremely thin metal films), all nonreflected light can be regarded as absorbed and by conservation of energy, the spectral directional absorptance can be found from

$$
A_{\lambda}^{\prime}\left(\Omega_{i}\right)=1-\rho_{\lambda}^{\prime}\left(\Omega_{i}\right) .
$$

Since there exists a direct correspondence between emittance and absorptance from Kirchhoff's law in thermodynamics, BRDF models in scattering theory can also be used to quantify the emissive properties of a material body.

\section{B. Gaussian random rough surfaces}

A 2D random rough surface $z=\xi(x, y)$ is commonly characterized by its height distribution function (HDF) and its autocovariance function (ACF), the former describing the surface height deviation from a mean surface level and the latter describing how the peaks and valleys are laterally distributed along the surface. In this paper, both the HDF $p_{h}(\xi)$ and the ACF $C(x, y)$ are assumed to have a Gaussian distribution, thus obeying the following properties:

$$
\begin{aligned}
& p_{h}(\xi)=\frac{1}{\sqrt{2 \pi \sigma^{2}}} \exp \left(-\frac{\xi^{2}}{2 \sigma^{2}}\right), \\
& C(x, y)=\exp \left(-\frac{x^{2}}{\tau_{x}^{2}}-\frac{y^{2}}{\tau_{y}^{2}}\right),
\end{aligned}
$$

where $\sigma$ is the rms height and $\tau_{x}$ and $\tau_{y}$ are the correlation lengths in $x$ and $y$, respectively. Thomas demonstrated that this Gaussian assumption is valid as long as the height at any point on the surface is not produced by a single one-off event (which is true of most surfaces). ${ }^{9}$ It is also assumed in this work that the surfaces are isotropic, i.e., showing no preferred roughness direction so that $\tau_{x}=\tau_{y}=\tau$.

As a result of the fact that both the HDF and the ACF are Gaussians, the slope distribution function $(\mathrm{SDF}) p_{s}(\xi)$ will also be a Gaussian, i.e.,

$$
p_{s}\left(\boldsymbol{\varsigma}_{x}, \boldsymbol{\varsigma}_{y}\right)=\frac{1}{2 \pi w_{x} w_{y}} \exp \left(-\frac{\boldsymbol{s}_{x}^{2}}{2 w_{x}^{2}}-\frac{\boldsymbol{s}_{y}^{2}}{2 w_{y}^{2}}\right),
$$

where $\boldsymbol{s}_{x}=\partial \xi / \partial x$ and $\boldsymbol{s}_{y}=\partial \xi / \partial y$ are the slopes in the $x$ and $y$ directions, respectively. The rms slopes are $w_{x}$ and $w_{y}$ and isotropy results in their equality so that $w_{x}=w_{y}=w$. It can be shown that the rms slope for a Gaussian random rough surface will be given by $w=\sqrt{2} \sigma / \tau .^{10}$ For simplicity, the factor of $\sqrt{2}$ will be omitted in the following treatment and when we speak of the rms slope or slope, we will only use the ratio $\sigma / \tau$.

For simulation purposes, the $2 \mathrm{D}$ random rough surfaces with the above properties can be generated by using the method outlined by Garcia and Stoll. ${ }^{11}$ Working on a discrete mesh of points in the $x-y$ plane, an uncorrelated Gaussian random rough surface distribution $\xi_{u}(x, y)$ is generated by using a Gaussian random number generator. To achieve correlation of surface points, this distribution is then convolved with a Gaussian filter

$$
F(x, y)=\frac{2}{\tau \sqrt{\pi}} \exp \left(-2\left(x^{2}+y^{2}\right) / \tau^{2}\right),
$$

which means that we set

$$
\xi(x, y)=\iint_{-\infty}^{\infty} F\left(x-x^{\prime}, y-y^{\prime}\right) \xi_{u}\left(x^{\prime}, y^{\prime}\right) d x^{\prime} d y^{\prime} .
$$

In practice, this can be implemented by using a fast Fourier transform (FFT) algorithm. Figure 2 shows two realizations of the 2D Gaussian random rough surfaces generated by using this method, for two different rms slopes. Figure 3 shows the statistical data for a surface generated with rms slope $\sigma / \tau=1.0$.

\section{The GO approximation}

The GO approximation is an approximation to the more exact numerical integration methods from electromagnetic wave theory. As an approximation, it is of course limited to certain roughness parameters, which have been discussed by Tang and co-workers. ${ }^{3,4}$ Although no rigorous study has been made in the region of validity of the $3 \mathrm{D}$ scattering approximation, the authors projected that the parameters derived for the $2 \mathrm{D}$ case would also very likely hold for the $3 \mathrm{D}$ case. ${ }^{4}$

The GO approximation is a ray-tracing approach, where energy bundles or rays are traced throughout their interactions with the surface until they leave it (see Fig. 4). The surface is assumed to be locally flat so that light is specularly reflected at each interaction point (this is known as the Fresnel approximation). Unlike many other approximations, the GO approximation treats the phenomena of multiple scattering and shadowing automatically. Multiple scattering describes the situation when light is scattered more than once from the surface (an example is given in Fig. 4 which shows double scattering), which becomes more important, the rougher the surface. Shadowing happens when some parts of the surface are blocked from the incident light due to obstruction from other sections (see the grayed areas in Fig. 4 
(a)

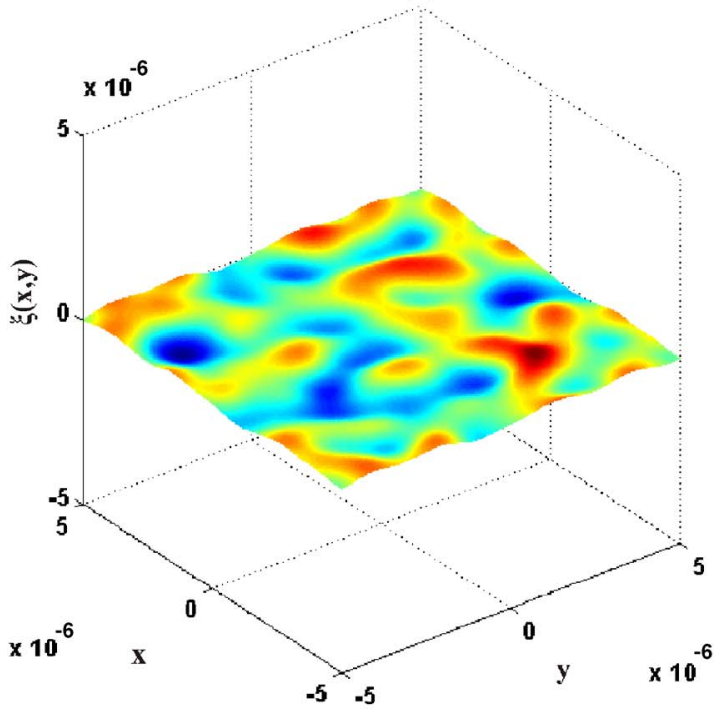

(b)

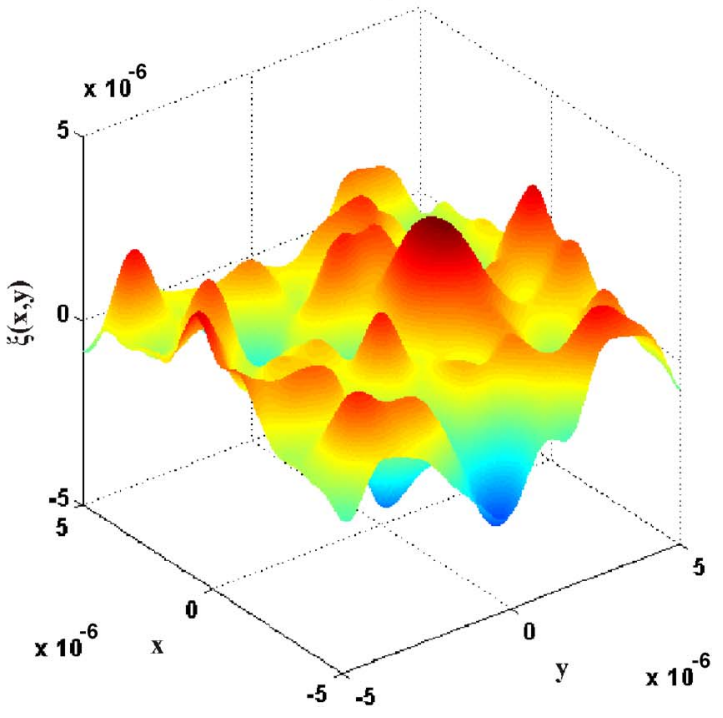

FIG. 2. (Color online) Two realizations of the $2 \mathrm{D}$ Gaussian random rough surfaces, with (a) $\sigma / \tau=0.1$ and (b) $\sigma / \tau=1.0$ (the correlation length $\tau$ was $1 \mu$ m in both cases).

for an illustration). Shadowing is important for oblique angles of incidence and becomes more important the larger the angle.

The first step of the ray-tracing technique consists of generating the Gaussian random rough surface for the prespecified correlation length, rms height, and surface length. The slopes and normals for all surface points are then estimated (see, for instance, Thürmer and Wüthrich ${ }^{12}$ ). The angles of incidence, the polar (zenith) angle $\theta_{0}$ and the azimuth angle $\varphi_{0}$, are then chosen and an incident ray vector is formed as $\overline{\mathbf{s}}_{i}=-\left[\sin \left(\theta_{0}\right) \cos \left(\varphi_{0}\right), \sin \left(\theta_{0}\right) \sin \left(\varphi_{0}\right), \cos \left(\theta_{0}\right)\right]$.

Next, a first reflection point is chosen. The first reflection points can be chosen from a random or an equally spaced distribution of points over the surface. The first reflection point chosen is checked for shadowing by analyzing all surface points in the direction of the incident ray (more specifically, the surface points lying in the plane formed by $\overline{\mathbf{s}}_{i}$ and the projection of $\overline{\mathbf{s}}_{i}$ onto the $x-y$ plane). If any surface point in this plane lies above the incident ray vector, the first reflection point is considered as shadowed and the next one is considered. If the reflection point is not shadowed, the direction of the reflected ray is calculated from Snell's law: $\overline{\mathbf{s}}_{r}$ $=\overline{\mathbf{s}}_{i}-2 \overline{\mathbf{n}}\left(\overline{\mathbf{s}}_{i} \cdot \overline{\mathbf{n}}\right)$, where $\overline{\mathbf{n}}$ is the local surface normal.

The energy of all of the incident rays $G_{i}$ are equally distributed but are compensated for different area projections at the first reflection points according to Lambert's cosine law. The energy of the reflected ray $G_{r}$ can essentially be found by multiplying the incident energy by the Fresnel coefficient $R_{s, p}\left(\theta_{0}, \varphi_{0}, n, k\right)$, which is found from Fresnel's equations and is dependent upon the incident polarization $(s$ or $p)$, the angles of incidence $\left(\theta_{0}, \varphi_{0}\right)$, and the optical constants of the medium (refractive index $n$ and extinction coefficient $k$ ). However, because of the random orientations of the local surface normal vectors, the incident polarization will change upon reflection, i.e., depolarization will occur. To account for this, the incident energy is decomposed into $s$ and $p$-polarized components $G_{i, s}$ and $G_{i, p}$, respectively.
These components are defined in a global reference frame defined by the $z$-axis unit vector $\overline{\mathbf{z}}$ and the direction of the incident ray $\overline{\mathbf{s}}_{i}$, which are the two vectors that define the global plane of incidence. In a similar manner, $\overline{\mathbf{z}}$ and the reflected ray $\overline{\mathbf{s}}_{r}$ will define the plane of reflection, where the polarized components of the reflected energy $G_{r, s}$ and $G_{r, p}$ are formed. The incident and reflected energies are then related according to

$$
\left[\begin{array}{c}
G_{r, s} \\
G_{r, p}
\end{array}\right]=\left[\begin{array}{ll}
\rho_{s s} & \rho_{p s} \\
\rho_{s p} & \rho_{p p}
\end{array}\right]\left[\begin{array}{c}
G_{i, s} \\
G_{i, p}
\end{array}\right],
$$

where $\rho_{s s}$ and $\rho_{p p}$ are the copolarized reflectivities and $\rho_{s p}$ and $\rho_{p s}$ are the cross-polarized reflectivities (the first and second index stand for the incidence and reflection, respectively).

The calculation of these polarized reflectivities involves two conversions of polarization components. First, the $s$ - and $p$-polarized components in global coordinates are transformed into their counterparts in the local frame of the specific interaction point (the frame defined by the local surface normal and incident ray vector). The local polarization components are multiplied by the Fresnel amplitude reflection coefficients and are then converted back into the global frame. Accordingly, the polarized reflectivities can be calculated from ${ }^{13,14}$

$$
\begin{aligned}
& \rho_{s s}=\left|\left(\overline{\mathbf{v}}_{r} \cdot \overline{\mathbf{s}}_{i}\right)\left(\overline{\mathbf{v}}_{i} \cdot \overline{\mathbf{s}}_{r}\right) r_{s}+\left(\overline{\mathbf{h}}_{r} \cdot \overline{\mathbf{s}}_{i}\right)\left(\overline{\mathbf{h}}_{i} \cdot \overline{\mathbf{s}}_{r}\right) r_{p}\right|^{2} /\left|\overline{\mathbf{s}}_{i} \times \overline{\mathbf{s}}_{r}\right|^{4}, \\
& \rho_{s p}=\left|\left(\overline{\mathbf{h}}_{r} \cdot \overline{\mathbf{s}}_{i}\right)\left(\overline{\mathbf{v}}_{i} \cdot \overline{\mathbf{s}}_{r}\right) r_{s}-\left(\overline{\mathbf{v}}_{r} \cdot \overline{\mathbf{s}}_{i}\right)\left(\overline{\mathbf{h}}_{i} \cdot \overline{\mathbf{s}}_{r}\right) r_{p}\right|^{2} /\left|\overline{\mathbf{s}}_{i} \times \overline{\mathbf{s}}_{r}\right|^{4}, \\
& \rho_{p s}=\left|\left(\overline{\mathbf{v}}_{r} \cdot \overline{\mathbf{s}}_{i}\right)\left(\overline{\mathbf{h}}_{i} \cdot \overline{\mathbf{s}}_{r}\right) r_{s}-\left(\overline{\mathbf{h}}_{r} \cdot \overline{\mathbf{s}}_{i}\right)\left(\overline{\mathbf{v}}_{i} \cdot \overline{\mathbf{s}}_{r}\right) r_{p}\right|^{2} /\left|\overline{\mathbf{s}}_{i} \times \overline{\mathbf{s}}_{r}\right|^{4}, \\
& \rho_{p p}=\left|\left(\overline{\mathbf{h}}_{r} \cdot \overline{\mathbf{s}}_{i}\right)\left(\overline{\mathbf{h}}_{i} \cdot \overline{\mathbf{s}}_{r}\right) r_{s}+\left(\overline{\mathbf{v}}_{r} \cdot \overline{\mathbf{s}}_{i}\right)\left(\overline{\mathbf{v}}_{i} \cdot \overline{\mathbf{s}}_{r r}\right) r_{p}\right|^{2} /\left|\overline{\mathbf{s}}_{i} \times \overline{\mathbf{s}}_{r}\right|^{4} .
\end{aligned}
$$

In Eq. (10), $r_{s}$ and $r_{p}$ are the Fresnel amplitude reflectivities (see, for instance, Modest ${ }^{8}$ ). $\overline{\mathbf{h}}$ represents the direc- 
(a)

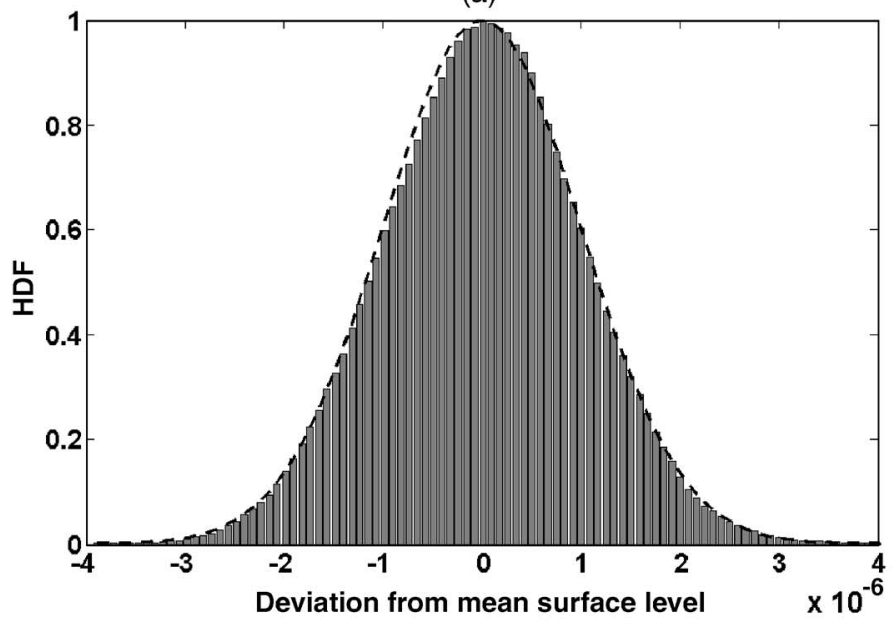

(b)

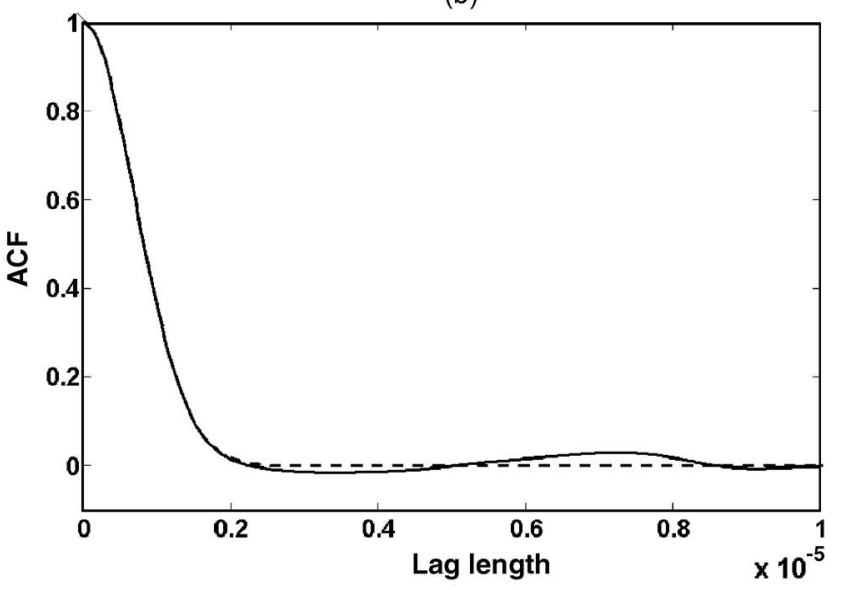

(c)

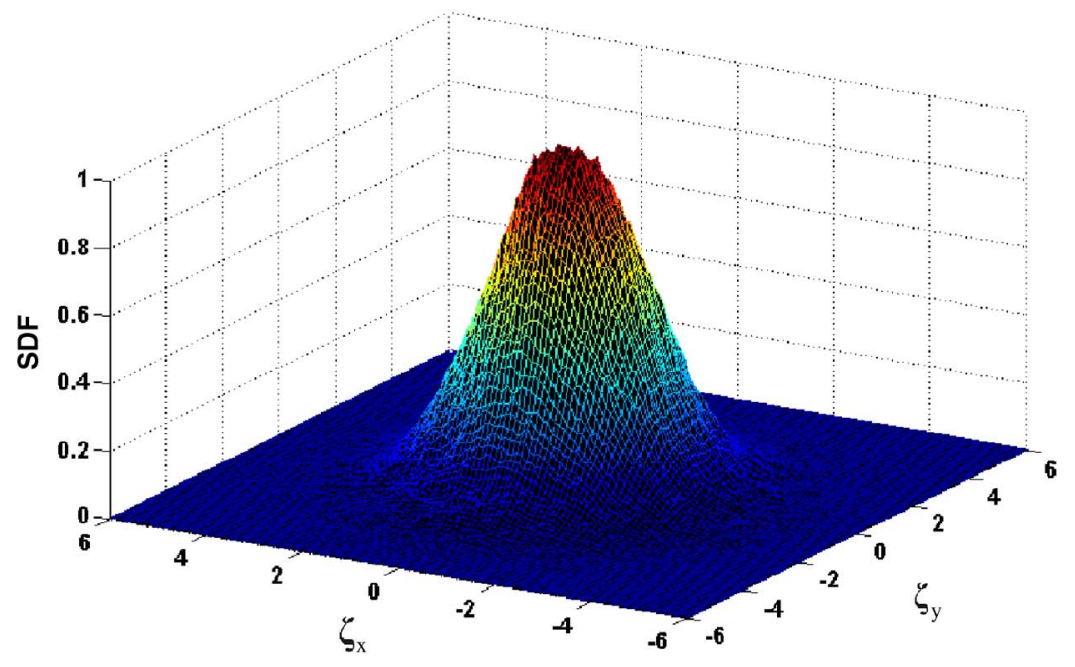

FIG. 3. (Color online) Surface statistics for a Gaussian random rough surface generated with $\sigma=1 \mu \mathrm{m}$ and $\tau=1 \mu \mathrm{m}$. (a) shows a normalized histogram of the HDF while (b) is a graph of the ACF, shown here in the $x$ direction as an example. In both figures, the exact Gaussians are included for reference as dashed lines. (c) shows the corresponding normalized 2D histogram of the SDF, which also follows a Gaussian distribution.

tion of the $s$ or horizontal polarized components and $\overline{\mathbf{v}}$ represents the direction of the $p$ or vertical polarized components, where incident vectors have subscripts $i$ and reflected vectors have subscripts $r$. Hence, the unit vectors $\overline{\mathbf{h}}_{i}$ and $\overline{\mathbf{v}}$ will be perpendicular and parallel to the plane of incidence, respectively, and similarly, $\overline{\mathbf{h}}_{r}$ and $\overline{\mathbf{v}}_{r}$ will be perpendicular and parallel to the plane of reflection, respectively. These vectors can be calculated from

$$
\begin{aligned}
& \overline{\mathbf{h}}_{i}=\frac{\overline{\mathbf{z}} \times \overline{\mathbf{s}}_{i}}{\left|\overline{\mathbf{z}} \times \overline{\mathbf{s}}_{i}\right|}, \quad \overline{\mathbf{v}}_{i}=\overline{\mathbf{h}}_{i} \times \overline{\mathbf{s}}_{i}, \\
& \overline{\mathbf{h}}_{r}=\frac{\overline{\mathbf{z}} \times \overline{\mathbf{s}}_{r}}{\left|\overline{\mathbf{z}} \times \overline{\mathbf{s}}_{r}\right|}, \quad \overline{\mathbf{v}}_{i}=\overline{\mathbf{h}}_{r} \times \overline{\mathbf{s}}_{r} .
\end{aligned}
$$

To determine whether the scattered ray $\overline{\mathbf{s}}_{r}$ strikes the surface again, a check similar to the one for shadowing is carried out. All of the surface points in the direction of $\overline{\mathbf{s}}_{r}$ are checked and if any surface point in the scattering plane (defined by $\overline{\mathbf{s}}_{r}$ and the projection of $\overline{\mathbf{s}}_{r}$ onto the $x-y$ plane) lies above $\overline{\mathbf{s}}_{r}$, then a new interaction point has been found and a new scattered ray is calculated. Once the scattering process has been completed, the remaining energy of the incident ray which finally leaves the surface is added to the BRDF.

When all of the first reflection points have been considered and all of the rays have been traced and scattered, the BRDF can be found from Eq. (1). The directionalhemispherical reflectance can then, in principle, be found from an integration over all of the scattered angles, as in Eq. (2), but can more easily and straight forwardly be found by dividing the sum of the energies of all of the rays, which leave the surface by the total incident energy. The absorptance is subsequently found from Eq. (3), i.e., by taking one minus the reflectance. To get statistically accurate results, the above procedure is then repeated for several realizations of surfaces having the same set of values for the correlation length and the rms height and an overall average can thus be calculated.

\section{RESULTS AND DISCUSSION}

The results presented below are the overall averages for each rms slope $\sigma / \tau$ taken from Monte Carlo simulations of 15-20 surface realizations (which was considered sufficient 


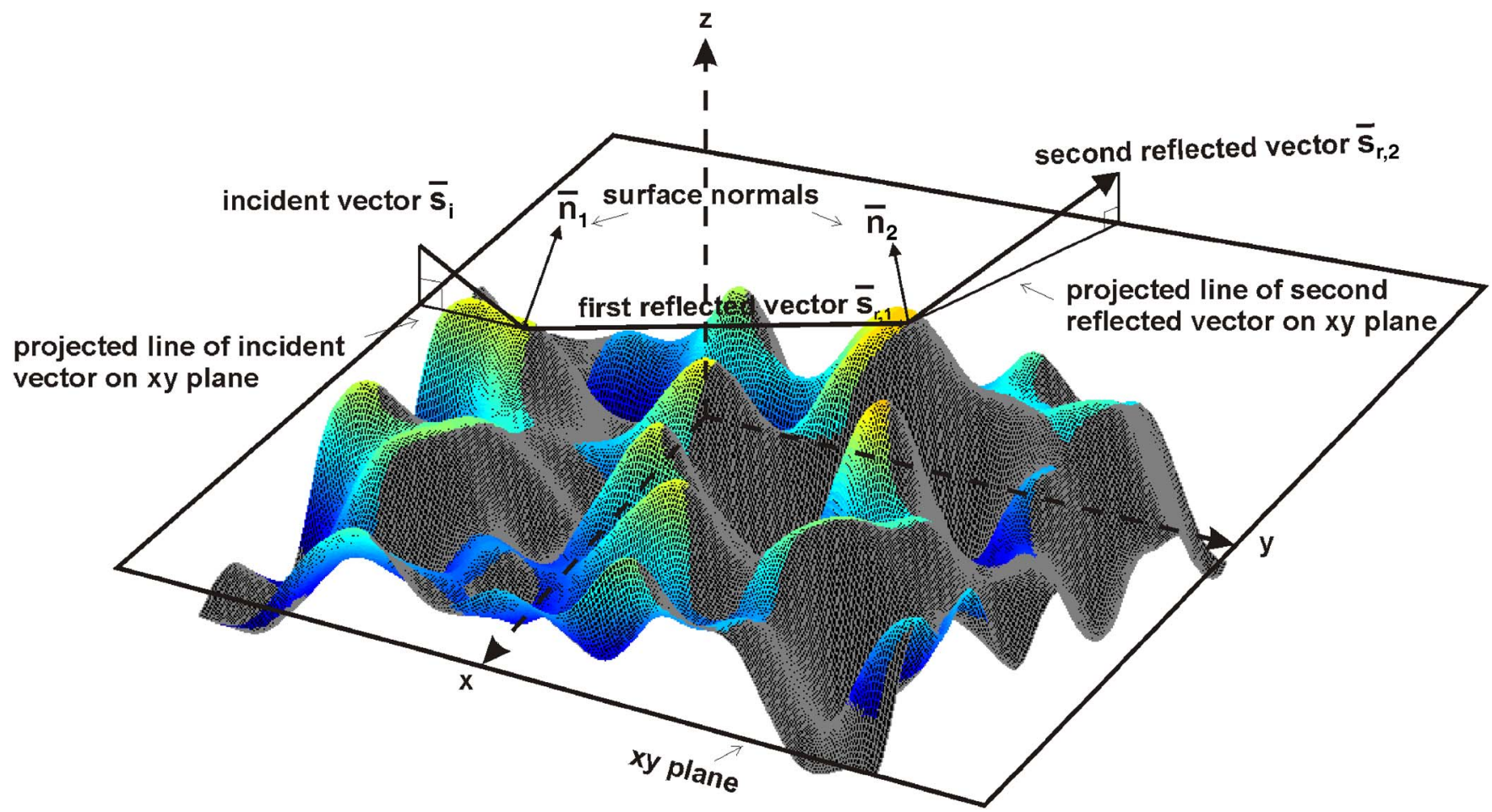

FIG. 4. (Color online) The geometry of the 3D ray tracing, which illustrates multiple (double) scattering and shadowing phenomena (shadowed regions are darker gray).

due to the small relative standard deviations involved, typically $1 \%$ or less) with sample sizes of $100 \times 100 \mu \mathrm{m}$.

The following analysis will initially be separated into normal and oblique incidences (Secs. III A and III B, respectively). Normal incidence is a somewhat special case because of the absence of shadowing but an important one, nonetheless, since this is the angle used in the initiation phase of most laser-material processing applications.

In Sec. III C, the predictions of Fresnel's equations and the Brewster angle are discussed in relation to rough surfaces. In Sec. III D, a comparison is made to some earlier published results from 2D ray-tracing simulations. Section III E concludes the results and discussion section with some general notes on roughness in the context of laser-materials processing.

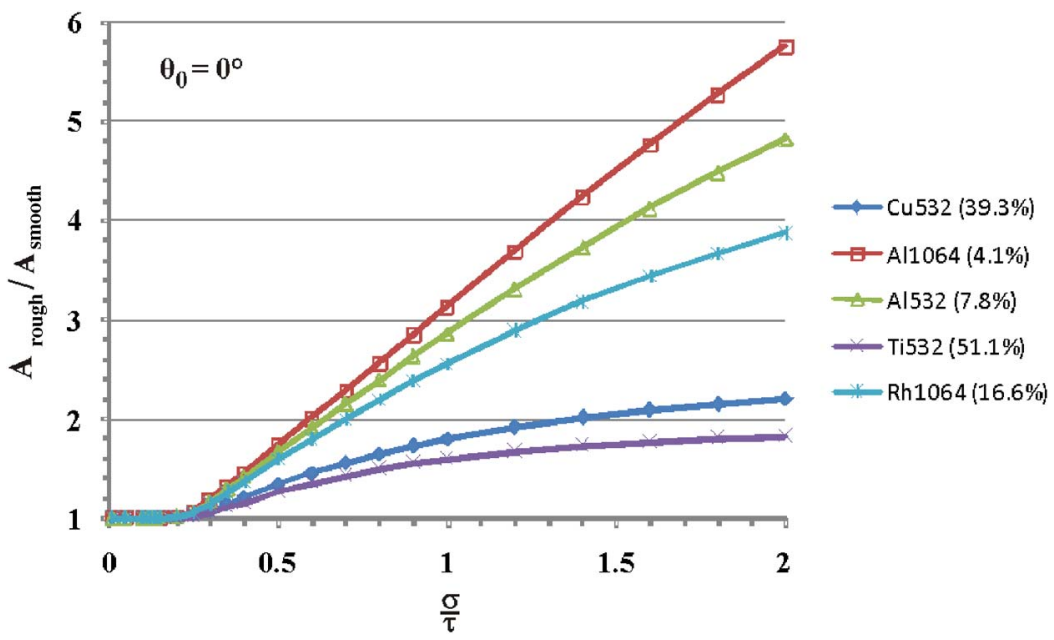

\section{A. Normal incidence $\theta_{0}=0^{\circ}$}

Figure 5 displays the absorptance results at normal incidence, i.e., when light is incident perpendicular to the mean surface plane. In the figure, the absorptance is plotted as a function of rms slope, after being normalized to the absorptance of a flat, smooth surface for the same metal/wavelength combination (i.e., the Fresnel absorptance at normal incidence). Two distinctly different regions can be identified.

(1) Roughness range of $0<\sigma / \tau<0.15$ and single scattering regime. The results show a minimal variation in absorptance over this roughness range. A comparison with Fig. 6 (see the blue line for normal incidence), which shows the average number of scattering points per ray as a function of slope, reveals this as a single scattering re-
FIG. 5. (Color online) Ratios of the absorptances of rough and smooth surfaces for normally incident light shown as a function of slope (roughness) for the metals listed in the legend (the number after the atomic symbol indicates the wavelength of the light involved). The smooth surface absorptances at normal incidence are given in parentheses. 


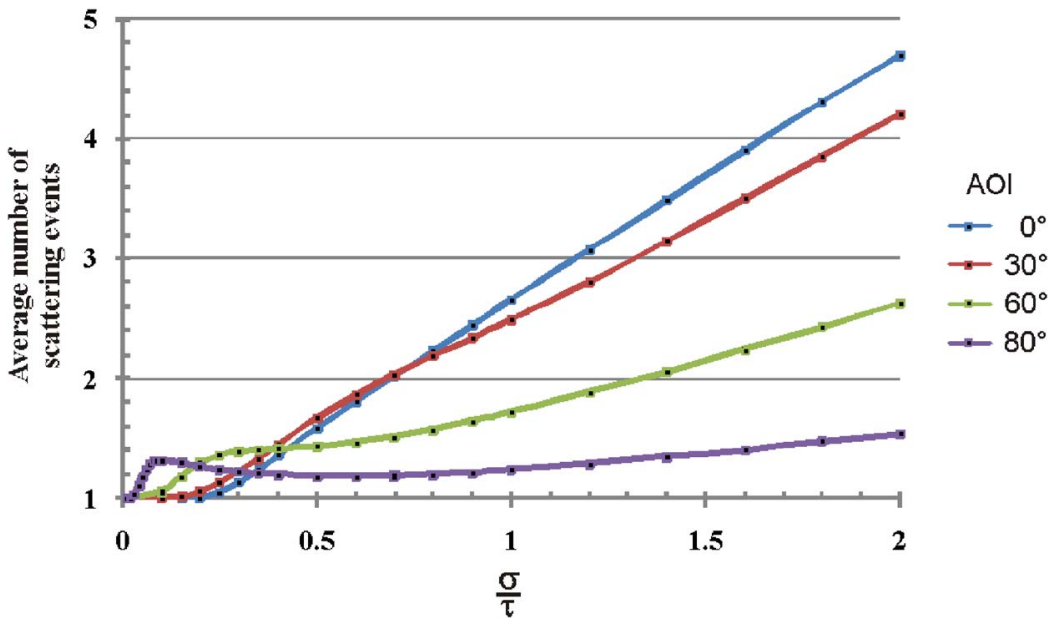

FIG. 6. (Color online) The average number of scattering events per incident ray as a function of the rms slope (roughness) $\sigma / \tau$ for the different angles of incidence in the study. gime where rays are scattered only once before leaving the surface, and thus, absorptance will only depend on the distribution of local surface normals and local angles of incidence. These angles are very small in this range of slopes, and hence, the absorptance shows no noticeable changes from the smooth surface value.

(2) Roughness range of $0.15<\sigma / \tau<2$ and multiple scattering regime. At around $\sigma / \tau \sim 0.15$, the absorptance starts to increase more sharply and a comparison with Fig. 6 explains that this is the threshold for double scattering. Beyond this threshold, the average number of scattering points increases almost linearly with slope. As each scattering point contributes energy to the surface, the absorptance will also increase in this slope range for all of the metal/wavelength combinations. However, as we can see in Fig. 5, less absorptive (i.e., more reflective) materials are found to be more sensitive to roughness than more absorptive ones. This is simply a consequence of "diminishing returns" where each new scattering point along a ray path contributes less and less energy for absorption due to the limited amount of energy available in the bundle. For example, if the absorptivity of a surface is $50 \%$, then the first scattering event will result in an absorption of $50 \%$, and the second will result in absorption of a further $25 \%$ (of the original energy), taking the total absorption level of up to $75 \%$. If, on the other hand, the absorptivity of the material is only $10 \%$, then the absorption figures from the primary and secondary scattering events will be $10 \%$ and $9 \%$-making a total of $19 \%$. This increase from $10 \%$ to $19 \%$ is considerably larger than the increase from $50 \%$ to $75 \%$ and this is the principle demonstrated in Fig. 5.

The practical consequence of this principle is that the deliberate roughening of a high reflectance material, such as aluminum at $1064 \mathrm{~nm}$, increases the absorption relatively more than it would for a material that already absorbs well in the smooth state (compare the relative increases in absorptance for aluminum and titanium in Fig. 5).

\section{B. Oblique incidence}

At oblique incidence, the analysis becomes more complex mainly due to the influence of shadowing. In the simulations, three different oblique angles of incidence were analyzed; $30^{\circ}, 60^{\circ}$, and $80^{\circ}$. At $30^{\circ}$ incidence, the results are very similar to those for normal incidence (compare Fig. 7 to Fig. 5), except for a small decrease in the double scattering threshold and slightly less overall scattering (and therefore

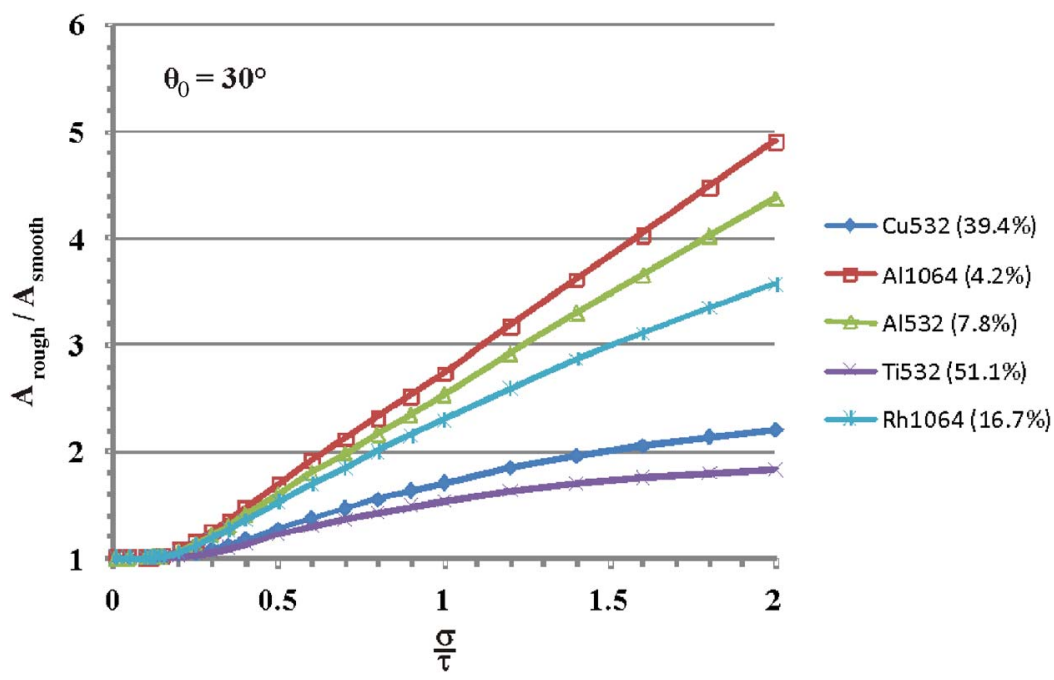

FIG. 7. (Color online) Ratios of the absorptances of rough and smooth surfaces for $\theta_{0}=30^{\circ}$ shown as a function of rms slope (roughness) for the metals listed in the legend (the number after the atomic symbol represents the wavelength of the light involved). The smooth surface absorptances at incidence angle $\theta_{0}=30^{\circ}$ are given in parentheses. 


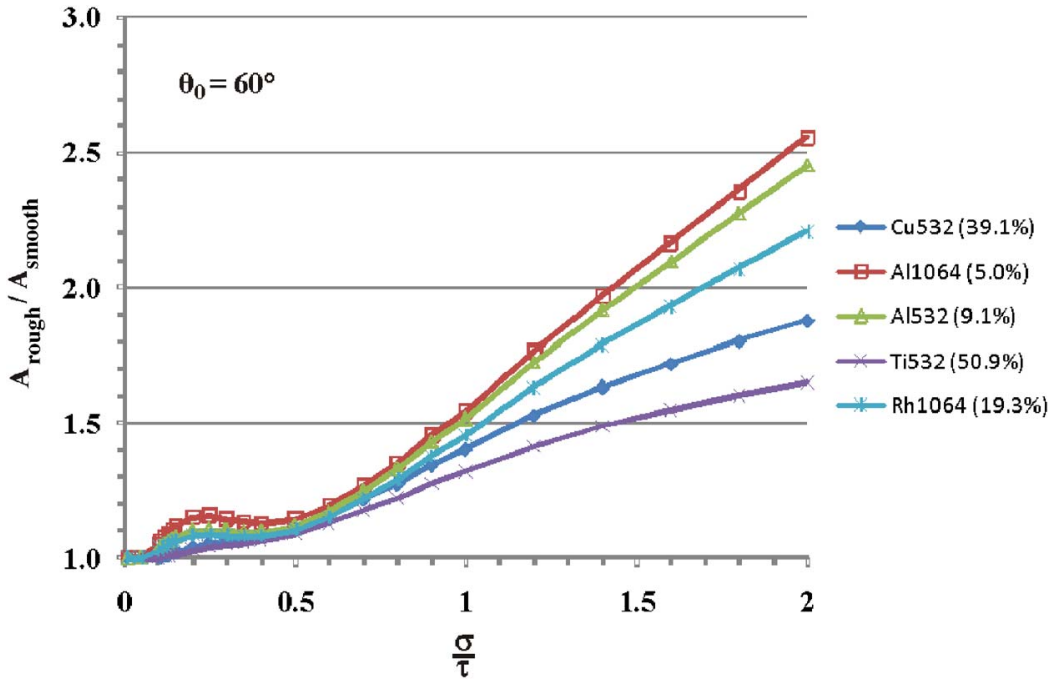

FIG. 8. (Color online) Ratios of the absorptances of rough and smooth surfaces for $\theta_{0}=60^{\circ}$ shown as a function of rms slope (roughness) for the metals listed in the legend (the number after the atomic symbol represents the wavelength of the light involved). The smooth surface absorptances at incidence angle $\theta_{0}=60^{\circ}$ are given in parentheses.

absorption) for the larger slopes (as seen in Fig. 6). These effects are even more apparent for $60^{\circ}$ (see Fig. 8), for which they will be discussed in greater detail.

At $60^{\circ}$ incidence, the analysis is most effectively treated by dividing the absorptance/roughness relationship into the following four segments.

(1) Roughness range of $0<\sigma / \tau<0.1$ and single scattering regime. As for normal incidence, this range of very small slopes involves only single scattering events and roughness, therefore, does not have a significant effect on the absorptance.

(2) Roughness range of $0.1<\sigma / \tau<0.2$ and multiple forward scattering regime.

A comparison of Figs. 8 and 7 reveals that the roughness threshold for double scattering reduces with increasing angle of incidence. This point is also confirmed by Fig. 6. This threshold reduction can be understood from Fig. 9. A simple condition for the introduction of double scattering can be placed forward as this; if the heights of the bumps on the sample surface are increased continuously from a flat state to the geometry shown in Fig. 9, double scattering will, in a first approximation, initially occur for surface patches where the first scattering point produces a horizontally reflected ray (i.e., a ray parallel to the mean reference plane). This corresponds to a ray with zenith angle of $90^{\circ}$ (the zenith

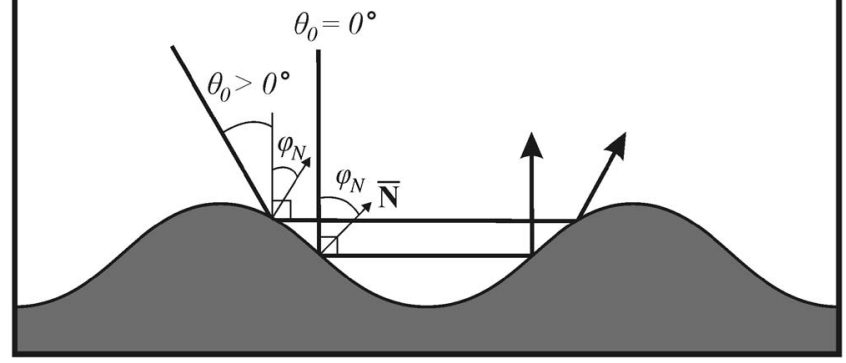

FIG. 9. The condition for double scattering is changed as the angle of incidence $\theta_{0}$ is increased since the limit of the surface inclination angle $\varphi_{N}$ required for generating a horizontally scattered ray is reduced. angle is the angle between the ray and the positive $z$ axis). At normal incidence, this occurs for surface patches where the normal vectors have inclination angles $\varphi_{N}=45^{\circ}$ (the inclination angle is the angle between the surface normal and the $z$ axis). As the angle of incidence increases, this inclination angle limit for double scattering decreases (as seen in the figure) according to $\varphi_{N}=\pi / 4-\theta_{0}$, so that, for instance, $\varphi_{N}\left(\theta_{0}\right.$ $\left.=30^{\circ}\right)=30^{\circ}, \varphi_{N}\left(\theta_{0}=60^{\circ}\right)=15^{\circ}$, and $\varphi_{N}\left(\theta_{0}=80^{\circ}\right)=5^{\circ}$.

Double scattering in this range involves the type of forward scattering events illustrated in Fig. 10(b), where the rays are scattered mainly in the forward direction with respect to the incident ray. These kinds of events will become more probable if the slope is higher, and thus, scattering and absorption will increase in this range.

(3) Roughness range of $0.2<\sigma / \tau<0.5$ and shadowinhibited regime. In this region, we find that the absorptance either levels out or decreases with increasing surface roughness. This contraintuitive result is explained as follows.

As the roughness of the surface is increased into this range, a considerable proportion of the surface becomes shadowed as a consequence of its topography and the high angle of incidence of the light. Figure 11 shows the average number of reflection points being shadowed and the phenomenon is demonstrated by Fig. 10(c). To understand the effect of shadowing on the absorptance, we need to refer back to Fig. 10(b). Here, we can see that the second order reflection at a high angle of incidence must involve the reflection of the ray of both sides of the same "valley." If the roughness is low, there will be no shadowing and the whole of both sides of any valley are available to take part in multiple absorption events (although not all primary reflections will give rise to the secondary ones). As the roughness is increased, shadowing becomes a feature and the sides of the valleys closest to the light source become increasingly unavailable as sites for primary reflections. This has the effect of inhibiting further increase in multiple forward scattering on this range (as seen in Fig. 6) and is the explanation for 
(a)

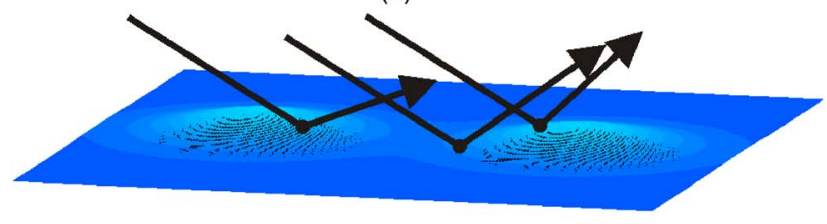

(b)

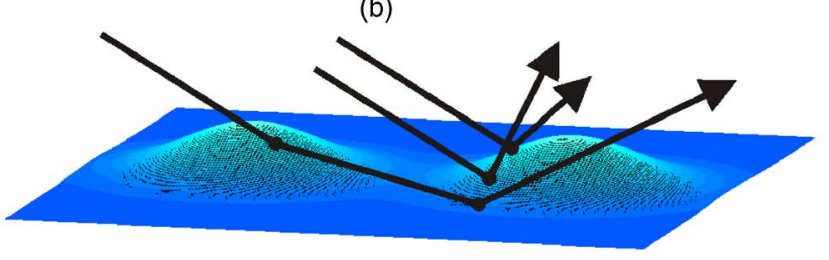

(c)

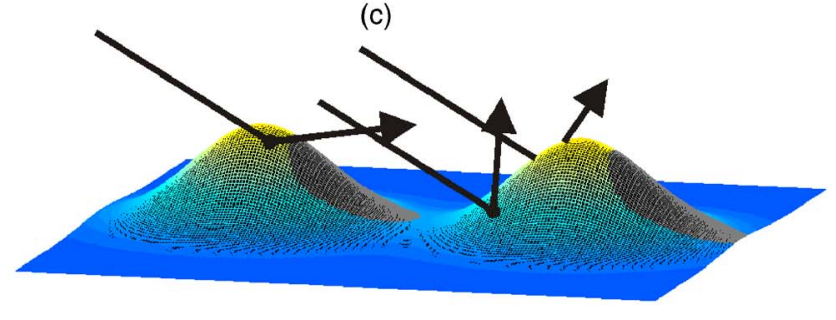

(d)

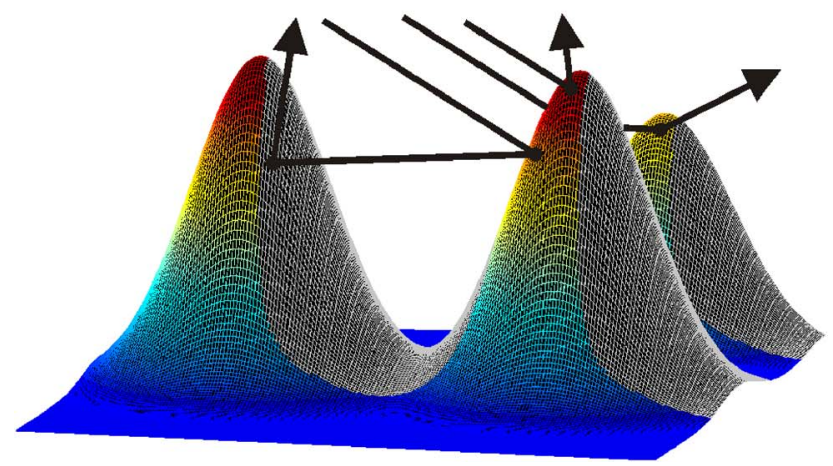

FIG. 10. (Color online) Four different regions of scattering behavior for light incident with a relatively large angle, illustrated here for $\theta_{0}=60^{\circ}$. (a) Very low roughness-single scattering only, (b) low roughness-multiple scattering in the forward direction is possible, (c) intermediate roughnessshadowing inhibits multiple scattering in the forward direction, and (d) high levels of roughness-multiple scattering in the backward and sideways directions.

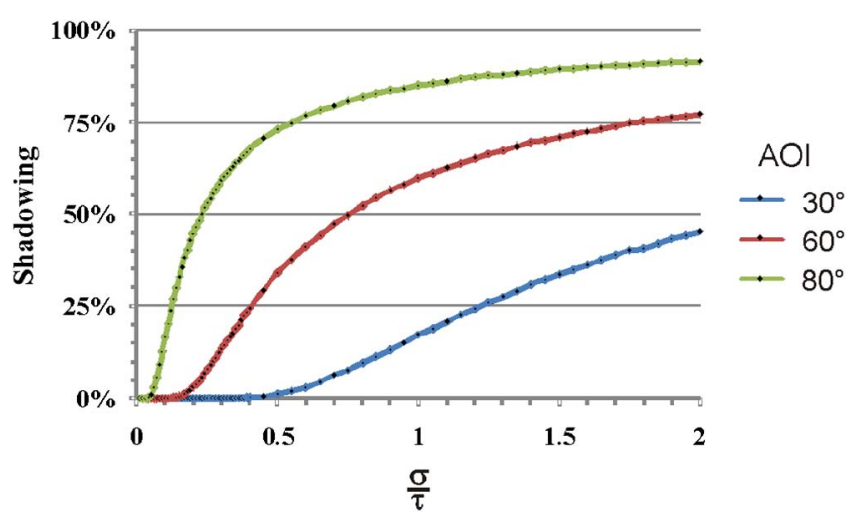

FIG. 11. (Color online) Shadowing, i.e., the average fraction of first reflection points being shadowed, as a function of rms slope.

the leveling out or reduction in absorptance which is visible in Fig. 8.

(4) Roughness range of $0.5<\sigma / \tau<2.0$ and multiple backand sidescattering regime. As the roughness is increased into this range, the absorptance is found to rise again. The effect of shadowing is, in this case, overcome by the type of higher order scattering events depicted in Fig. 10(d), where rays are scattered laterally (sideways) as well as in the backward direction (backscattering). These events make the shadowed regions once again available for scattering and absorption, which leads to an increase in absorptance.

For the grazing angle of incidence $80^{\circ}$ (see the results in Fig. 12), we divide the roughness range into the following two regions.

(1) Roughness range of $0<\sigma / \tau<0.5$ and shadow-inhibited single scattering regime. The strong influence of shadowing (see the $80^{\circ}$ line in Fig. 11) in this region inhibits multiple scattering to the extent that the absorptance is largely determined by single scattering events, as seen in Figs. 13(a)-13(c) (and Fig. 6 which shows the low level of scattering) involved. As single scattering is the dominant interaction, the absorptance will therefore mainly be a function of the distribution of local angles of incidence (as was the case for the single scattering, low

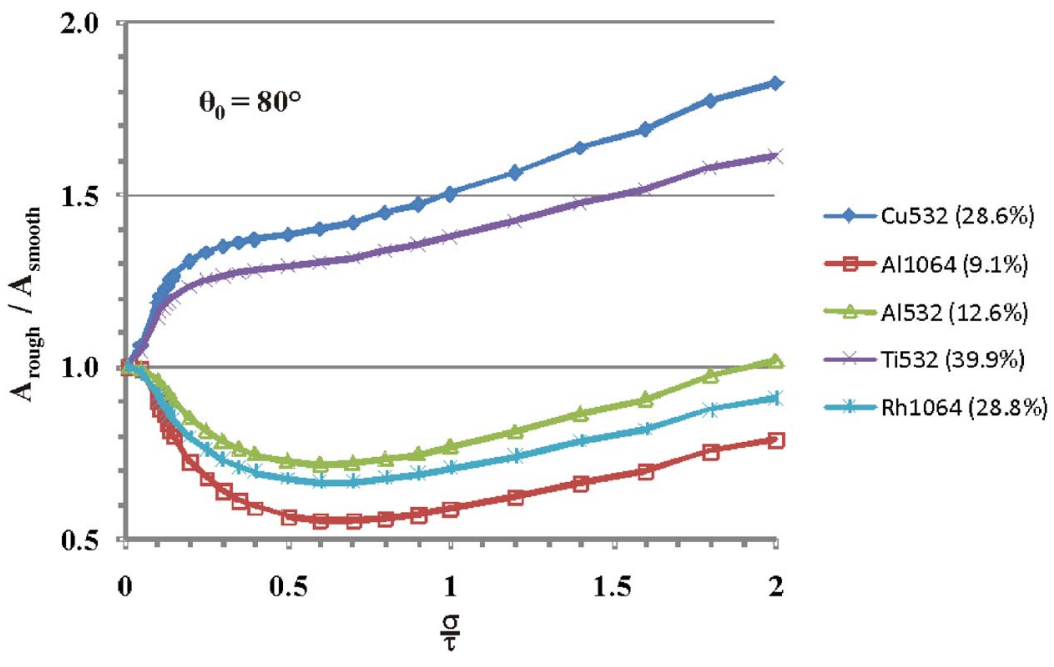

FIG. 12. (Color online) Ratios of the absorptances of rough and smooth surfaces for $\theta_{0}=80^{\circ}$ shown as a function of rms slope (roughness) for the metals listed in the legend (the number after the atomic symbol represents the wavelength of the light involved). The smooth surface absorptances at incidence angle $\theta_{0}=60^{\circ}$ are given in parentheses. 
(a)
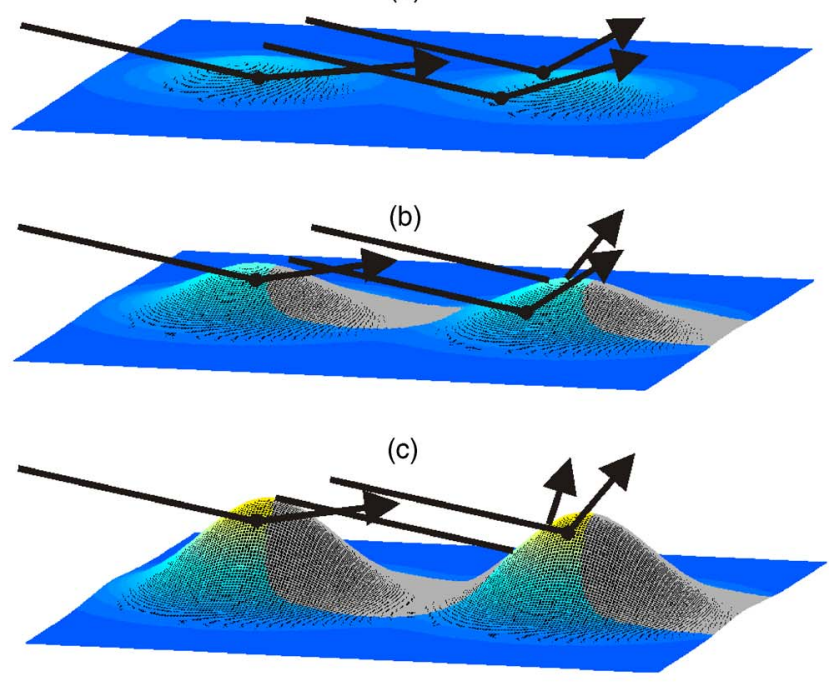

(d)

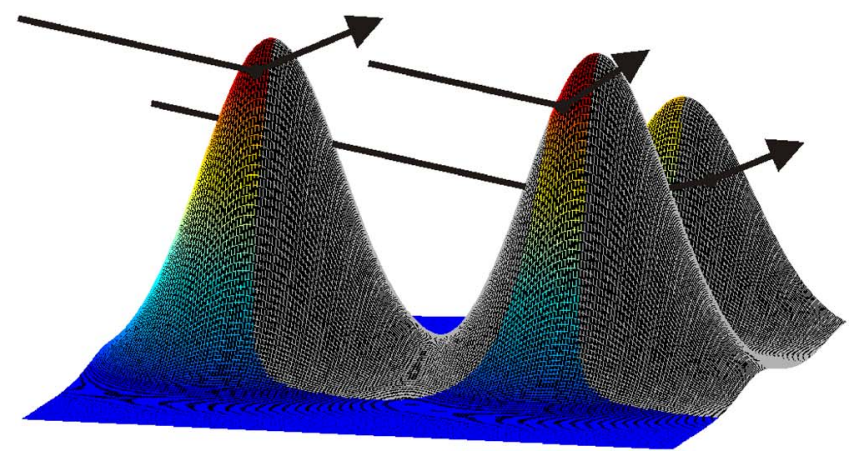

FIG. 13. (Color online) Four different regions of scattering behavior for light incident with a relatively large angle, illustrated here for $\theta_{0}=60^{\circ}$. (a) Very low roughness - single scattering only, (b) low roughness-single scattering remains the dominant interaction due to shadowing, (c) intermediate roughness - shadowing continues to inhibit multiple forward scattering events, and (d) high levels of roughness-multiple scattering is strongly inhibited in the forward and backward directions but may occur laterally as seen by the nonshadowed regions along the sides of the hills.

roughness regimes discussed earlier).

The results in Fig. 12 show a clear division between metal/wavelength combinations for which the absorptance increases with rms slope and combinations for which it does not. The determining factors here are: a) where the Brewster angle of the metal/wavelength is situated relative to the global angle of incidence and b) the shape of the Fresnel (angle of incidence/absorption) curve for the particular metal/wavelength combination. Figure 14 shows how the average local angle of incidence changes with slope (roughness) while Fig. 15 shows the Fresnel absorptances of the different metal/ wavelengths in the study. Since the average local angle of incidence experienced by the incident rays decreases with increasing roughness from $80^{\circ}$ to approximately $50^{\circ}$, we can see from Fig. 15 that the absorptance of aluminum at 1064 and $532 \mathrm{~nm}$ as well as of rhodium at $1064 \mathrm{~nm}$ will decrease with roughness, while titanium, gold, and copper at $532 \mathrm{~nm}$ will increase, which is what the results of the simulations in Fig. 12 suggest.

(2) Roughness range of $\sigma / \tau>0.5$ and multiple sidescatter-

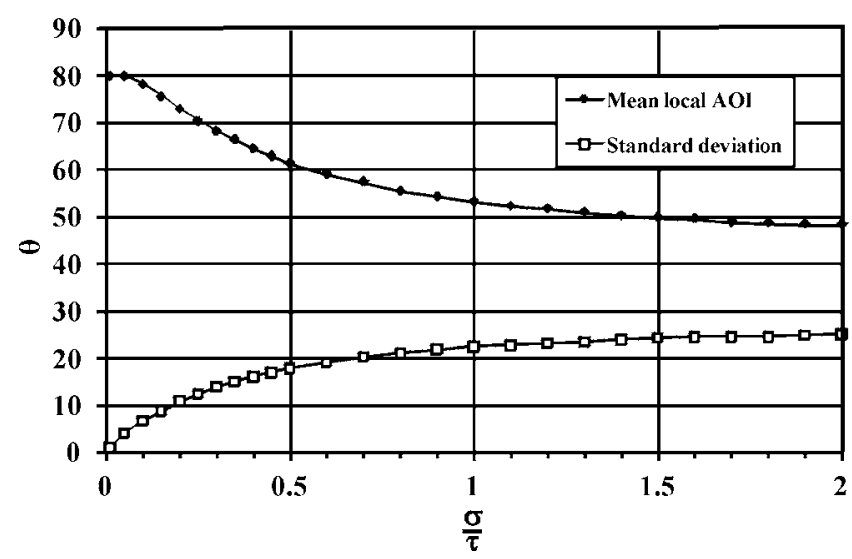

FIG. 14. The average local angle of incidence as a function of rms slope for rays incident with a global angle of incidence $\theta_{0}=60^{\circ}$.

ing regime. For the larger slopes, e.g., for $\sigma / \tau>0.5$, backscattering is strongly suppressed because of the geometry and it is mainly sidescattering which causes a moderate increase in the absorptance in this range.

\section{Fresnel absorptance and the Brewster angle for rough surfaces}

In some processing applications, such as laser cleaning and laser hardening (as well as other surface treatment processes), it is believed that a laser applied at or close to the Brewster angle of incidence can be beneficial for maximizing absorption. The Brewster angle is an angle usually situated in the range of $60^{\circ}-85^{\circ}$ (see Fig. 15 for a few examples) that can be found from Fresnel's equations. However, Fresnel's equations are defined for a perfectly flat and smooth surface and cannot be expected to hold for rougher surfaces. Figures 16 and 17 show the simulation results for the angular dependence of the absorptance for aluminum at $1064 \mathrm{~nm}$ and for copper at $532 \mathrm{~nm}$, respectively. As the roughness is increased from an almost smooth state $\sigma / \tau=0.1$ to a medium rough state $\sigma / \tau=0.3$, the Brewster maximum is reduced and eventually disappears for aluminum [see Fig. 16(a)] and for both aluminum and copper, the Fresnel predictions are replaced by a more flat angular

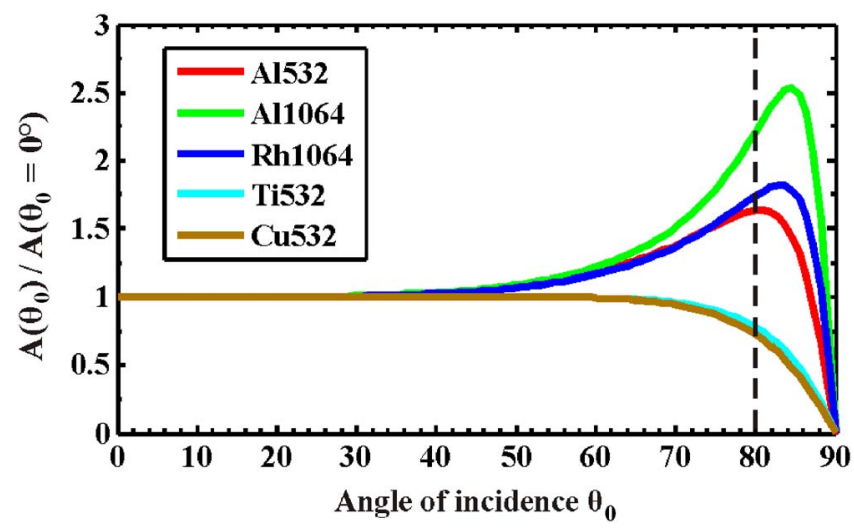

FIG. 15. (Color online) Fresnel absorptances of the metal/wavelengths in the study, normalized to the value at normal incidence $\left(\theta_{0}=0^{\circ}\right)$. The Fresnel absorptance at $80^{\circ}$ is indicated in the figure. 
(a)

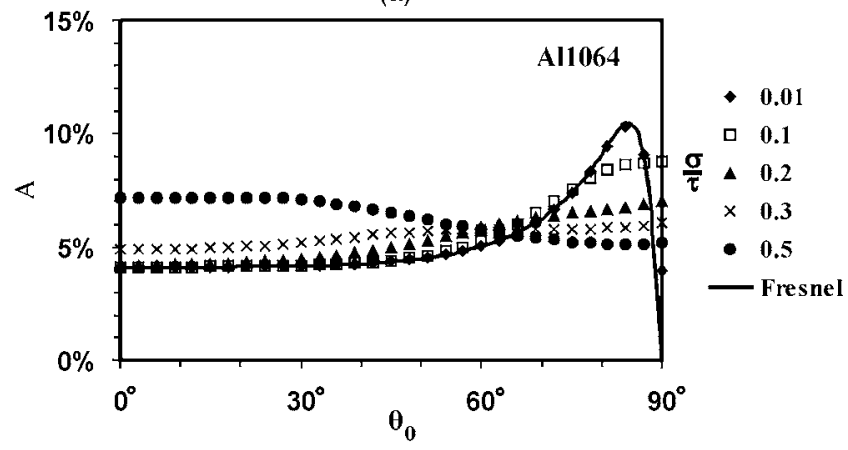

(b)

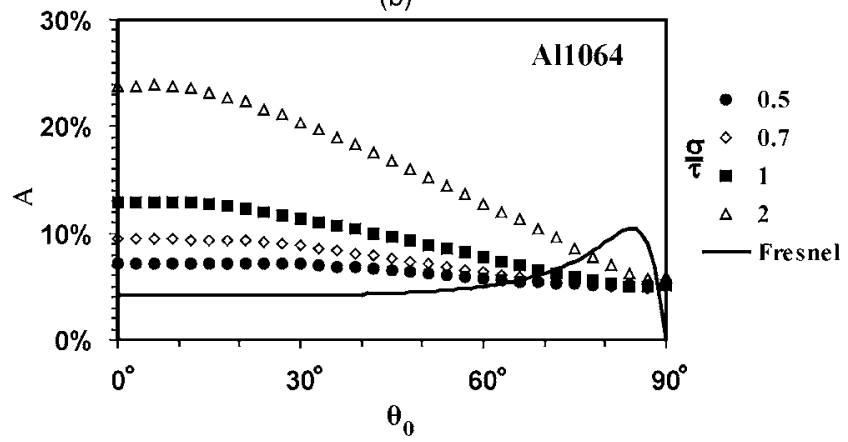

FIG. 16. The absorptance vs angle of incidence for aluminum at $\lambda$ $=1064 \mathrm{~nm}$, for a range of different rms slopes (roughness). In (a), $A(\theta)$ is shown for the range of small and medium slopes where $0.01 \leq \sigma / \tau \leq 0.5$, while in (b), it is given for the range of larger slopes where $0.5 \leq \sigma / \tau$ $\leq 2.0$. The Fresnel absorptance curve has been included for reference.

response [see Figs. 16(a) and 17(a), respectively]. As roughness is increased further to rough and very rough surfaces, a new maximum is formed at or close to normal incidence, as Figs. 16(b) and 17(b) show.

\section{Comparison between $2 \mathrm{D}$ and 3D ray-tracing models}

In a previous publication by the present authors, ${ }^{2}$ a $2 \mathrm{D}$ ray-tracing model was developed to study the effects of surface roughness on light absorption. This $2 \mathrm{D}$ model had a limited application to real surfaces and this is the main reason for the extension of the work to the isotropic 3D model surface shown in Fig. 2. However, the rippled liquid surface inside a laser cutting zone is one type of topography which may be better understood by reference to the $2 \mathrm{D}$ work. Figure 9 is typical of the figures produced in the 2D model because it involves no Y component to the light-surface interaction. Results from this type of $2 \mathrm{D}$ analysis can be directly used for real surfaces of the type shown in Fig. 18 (i.e., a real surface covered in parallel ripples). This surface is closer to the topography of the liquid in a laser cutting melt than the isotropic surface described in Fig. 2, and thus, in this case, the 2D model may be more useful as a starting point. One other area of application of the 2D model may be in the absorption of light by a surface which has been ground or machined with parallel grooves.

Although the 2D and 3D models give the same phenomenological results, they exhibit quantitative differences, as shown in Fig. 19. This figure shows the average number of scattering points for the four different angles analyzed in the (a)

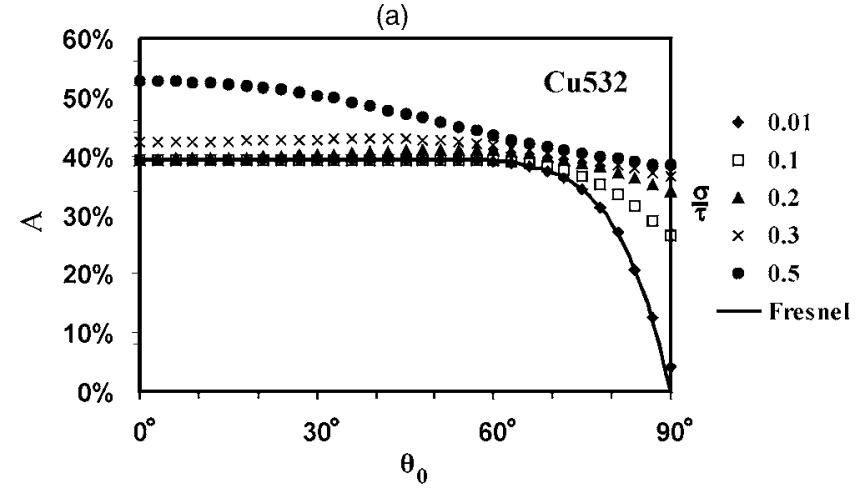

(b)

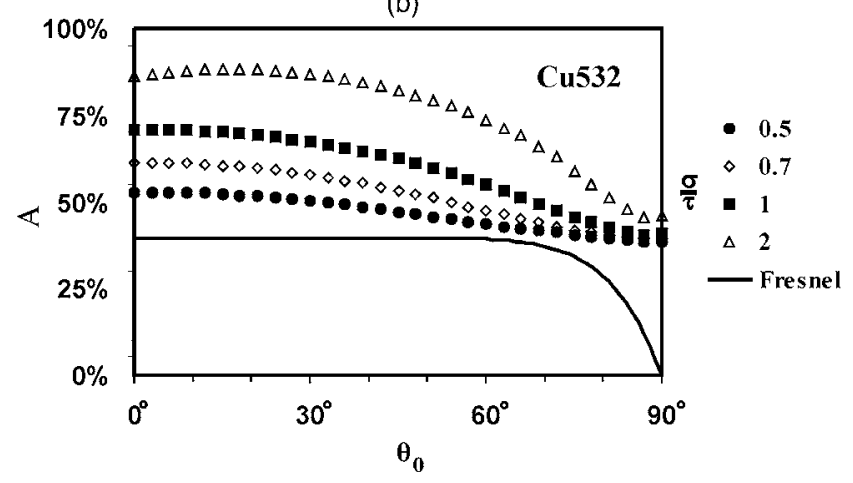

FIG. 17. The absorptance vs angle of incidence for copper at $\lambda=532 \mathrm{~nm}$, for a range of different rms slopes (roughness). In (a), $A(\theta)$ is shown for the range of small and medium slopes where $0.01 \leq \sigma / \tau \leq 0.5$, while in (b), it is given for the range of larger slopes where $0.5 \leq \sigma / \tau \leq 2.0$. The Fresnel absorptance curve has been included for reference.

two ray-tracing models. In all of the cases the overall behavior is very similar but the level of scattering is progressively higher in the $3 \mathrm{D}$ modeling case for surfaces above a certain roughness (slope) threshold.

The main reason for the increased level of scattering in $3 \mathrm{D}$ modeling as compared to the $2 \mathrm{D}$ modeling case can easily be appreciated by comparison of Figs. 18 and 2. Figure 2 describes the type of surface modeled in the 3D model and Fig. 18 is an equivalent description of the 2D model surface. It is clear that there are "hills and valleys" in all of the directions in the case of Fig. 2 whereas the surface only undulates in one direction in Fig. 18. This means that in the 3D model case (Fig. 2), there are more slopes available to

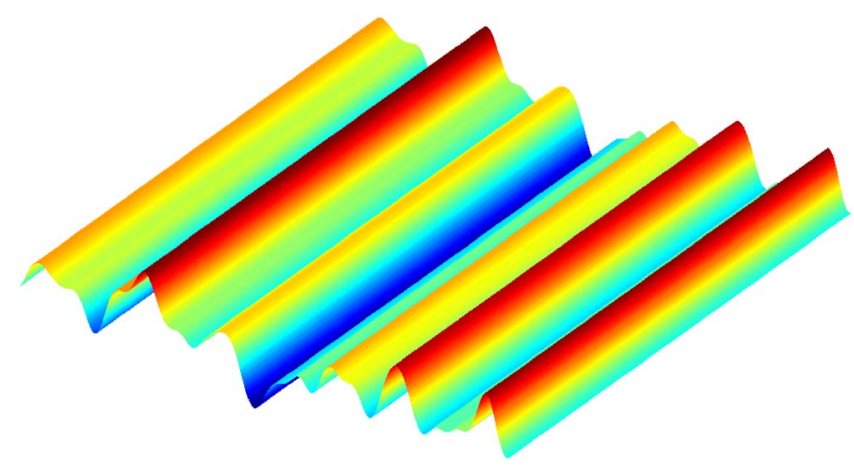

FIG. 18. (Color online) The type of surface (covered in parallel ripples or grooves) which may be better analyzed by using the $2 \mathrm{D}$ rather than the $3 \mathrm{D}$ model. 
(a)

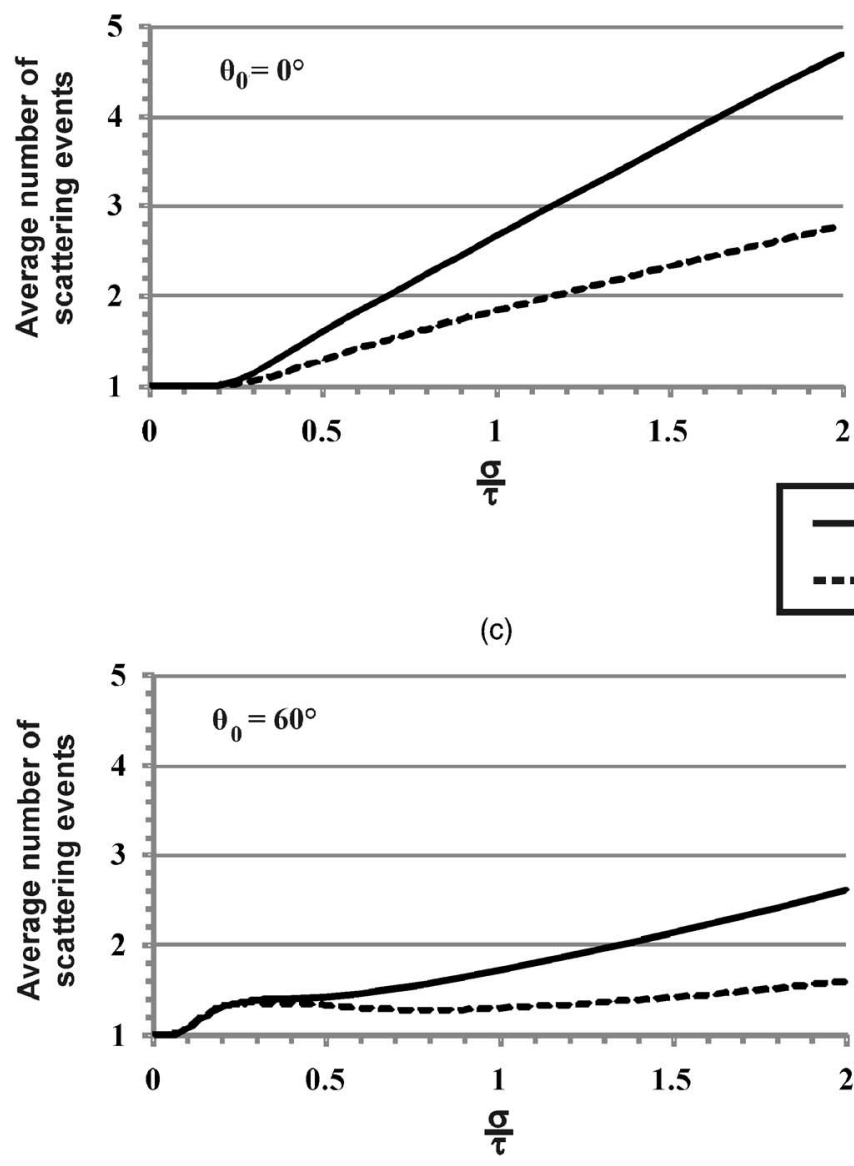

(b)

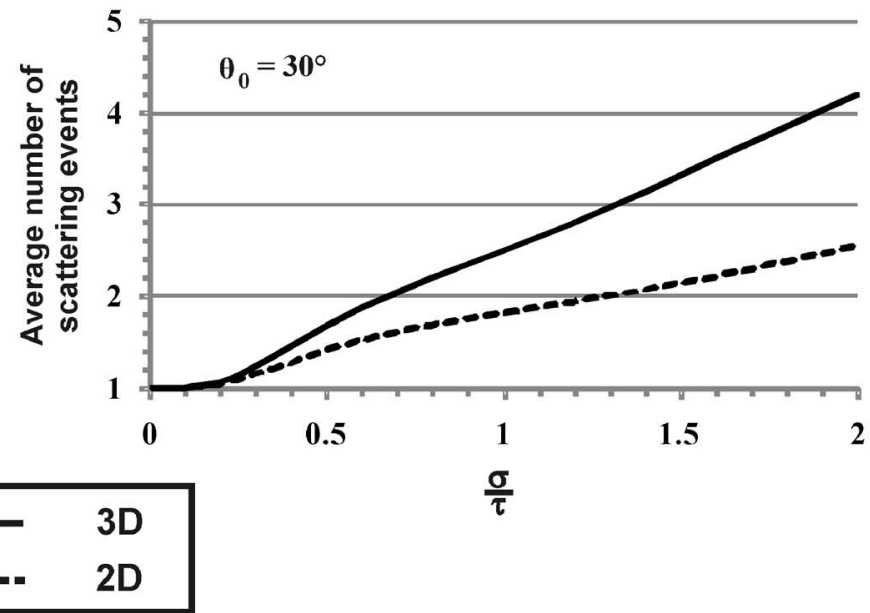

(d)

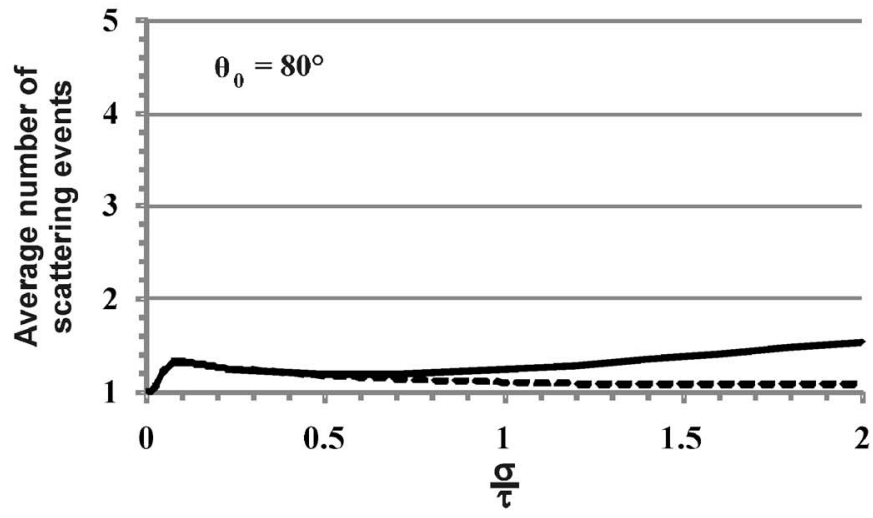

FIG. 19. Level of scattering in the 2D and 3D ray-tracing models. The results are from the simulations presented in this paper as well as from a previous one (Ref. 2).

scatter the incident light rays downward. This improved efficiency of the isotropic surface to scatter light downward results in an increase in the level of multiple scattering in the 3D model results, as shown in Fig. 19. This increase in multiple scattering will of course be accompanied by an increase in absorptance.

\section{E. Surface roughness in the context of laser- materials processing}

The results of this work have revealed a number of relationships between roughness and absorptance which should be of use to workers in the field of laser-materials processing. However, it should be appreciated that simply measuring the roughness in terms of rms height (or $\mathrm{Ra}$ ) is inadequate for estimations of absorptance. This commonly used measure of roughness gives no information about the crowdedness of the hills and valleys which go to make up the material surface roughness. Thus, there is no information about the rms slope associated with the surface roughness, which has been demonstrated in this paper to be a major influence on absorptance.

\section{CONCLUSIONS}

In this paper, a 3D ray tracing of the 2D Gaussian random rough surfaces has been used to study the effects of roughness on laser absorption. Although the investigation has been focused on specific metals (aluminum, copper, gold, rhodium, and titanium) at the particular wavelengths of the Nd:YAG (yttrium aluminum garnet) laser (1064 and 532 $\mathrm{nm}$ ), the authors feel that the phenomenological results and conclusions should be valid over a much wider range as long as the simulation parameters (wavelengths, angles of incidence, rms heights and correlation lengths) are within the confines of the validity region of the GO approximation as shown by Tang et al. ${ }^{3}$

For light incident, normally $\left(0^{\circ}\right)$ or with a relatively small angle $\left(30^{\circ}\right)$, the laser absorptance was found to increase with roughness (rms slope) after the threshold for multiple (double) scattering had been reached. The increase in absorptance with roughness is most pronounced for metals which are very reflective in the flat, smooth state.

For light incident at relatively large angles of incidence $\left(60^{\circ}\right.$ in the case of these results), the threshold for multiple scattering is lowered. After the threshold, the absorptance first increases with roughness (rms slope) but then levels out or even decreases in a medium roughness range where shadowing inhibits multiple scattering. As the surface roughness is further increased, the absorptance rises again due to higher order scattering events which make shadowed regions once again available for absorption.

For grazing incidence $\left(80^{\circ}\right.$ in the case of these results), 
single scattering dominates the behavior for surfaces with small and medium roughness (rms slope). The absorptance increases or decreases with increasing roughness depending on the location of the Brewster angle of the particular metal/ wavelength combination involved. For very rough surfaces, the absorptance increases with roughness due to the introduction of higher order scattering events.

In a study of the full angular dependence of absorption, it was found that the Fresnel angle of dependency only was satisfied for very smooth surfaces (with very small rms slopes). For medium rough surfaces, the Brewster maximum is suppressed and there is a flatter angular response. For very rough surfaces, there is a maximum at or close to normal incidence.

It was also found that a previous 2D model of ray tracing described similar phenomena to the present 3D model but predicted generally lower levels of multiple scattering and, therefore, absorption. The 2D model could be useful, however, in situations where the laser is impingent on a rippled or nonisotropic machined surface.

Finally, this paper has highlighted the point that any roughness index, which relies only on height measurements, is of little use when analyzing the absorptance of light by a surface. For the purpose of this type of analysis, it is important to know the rms slope which is typical of the surface in question.

${ }^{1}$ D. Bergström and A. F. H. Kaplan, The 16th Meeting on Mathematical Modelling of Materials Processing with Lasers, January 2003 (Igls, Austria, 2003).

${ }^{2}$ D. Bergström, J. Powell, and A. F. H. Kaplan, J. Appl. Phys. 101, 113504 (2007).

${ }^{3}$ K. Tang, R. Dimenna, and R. Buckius, Int. J. Heat Mass Transfer 40, 49 (1996).

${ }^{4}$ K. Tang and R. Buckius, Int. J. Heat Mass Transfer 41, 2037 (1998).

${ }^{5}$ K. Tang, P. Kawka, and R. Buckius, J. Thermophys. Heat Transfer 13, 169 (1999).

${ }^{6}$ P. Schott, N. de Beaucoudrey, and C. Bourlier, Int. J. Remote Sens. 7, 4214 (2003).

${ }^{7}$ Q. Zhu, Ph.D. thesis, Georgia Institute of Technology, 2004.

${ }^{8}$ M. Modest, Radiative Heat Transfer (Academic, San Diego, 2003).

${ }^{9}$ T. Thomas, Rough Surfaces (Longman, New York, 1982).

${ }^{10}$ L. Tsang, J. A. Kong, and K.-H. Ding, Scattering of Electromagnetic Waves: Theories and Applications (Wiley, New York, 2000).

${ }^{11}$ N. Garcia and E. Stoll, Phys. Rev. Lett. 52, 1798 (1984).

${ }^{12}$ G. Thürmer and C. Wüthrich, Comput. Graph. Forum 16, C15 (1997).

${ }^{13}$ L. Tsang and J. A. Kong, J. Appl. Phys. 51, 673 (1980).

${ }^{14}$ H. Lee, Ph.D. thesis, Georgia Institute of Technology, 2006. 